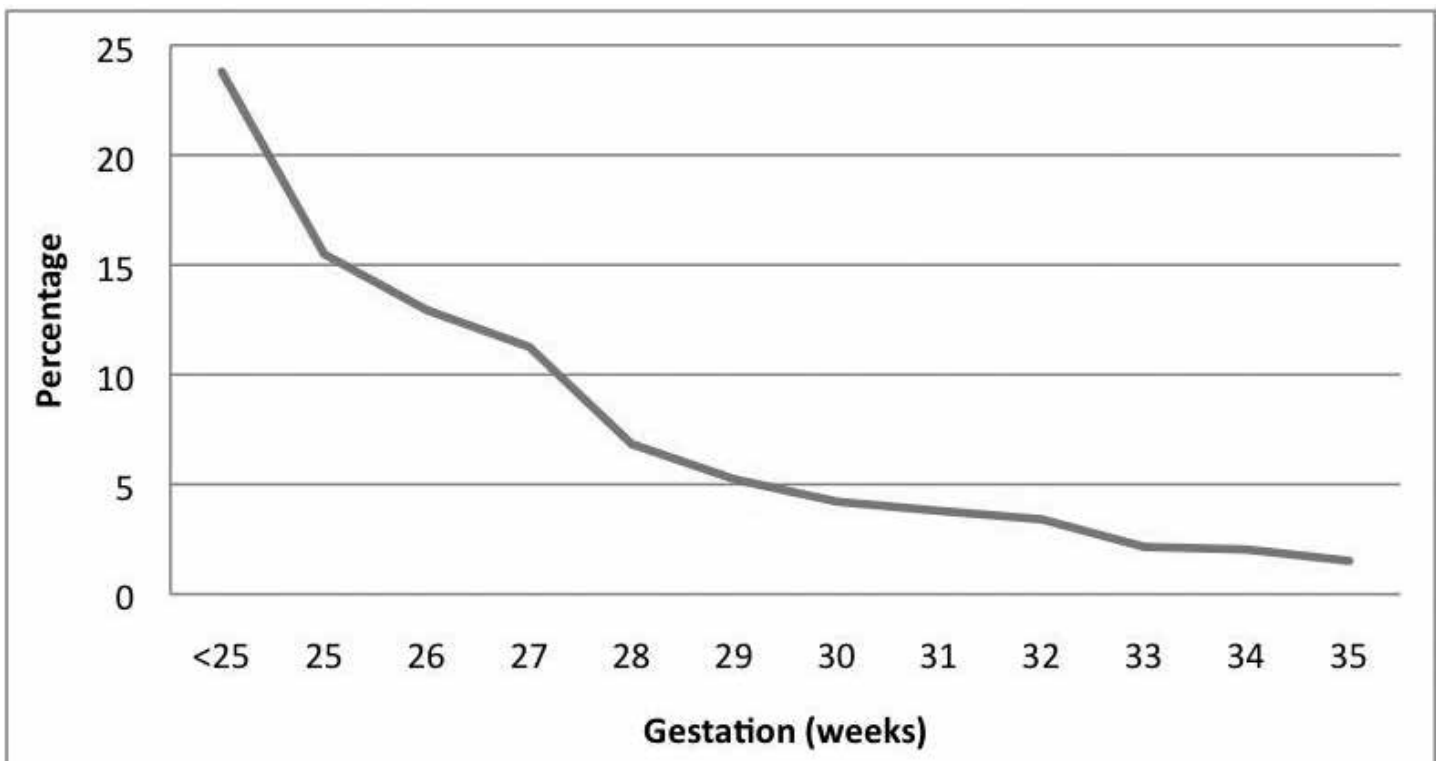

Abstract G50 Figure 2 Percentage of ex-premature babies alive at day 28 of life who are admitted to PICU before their second birthday by gestation.

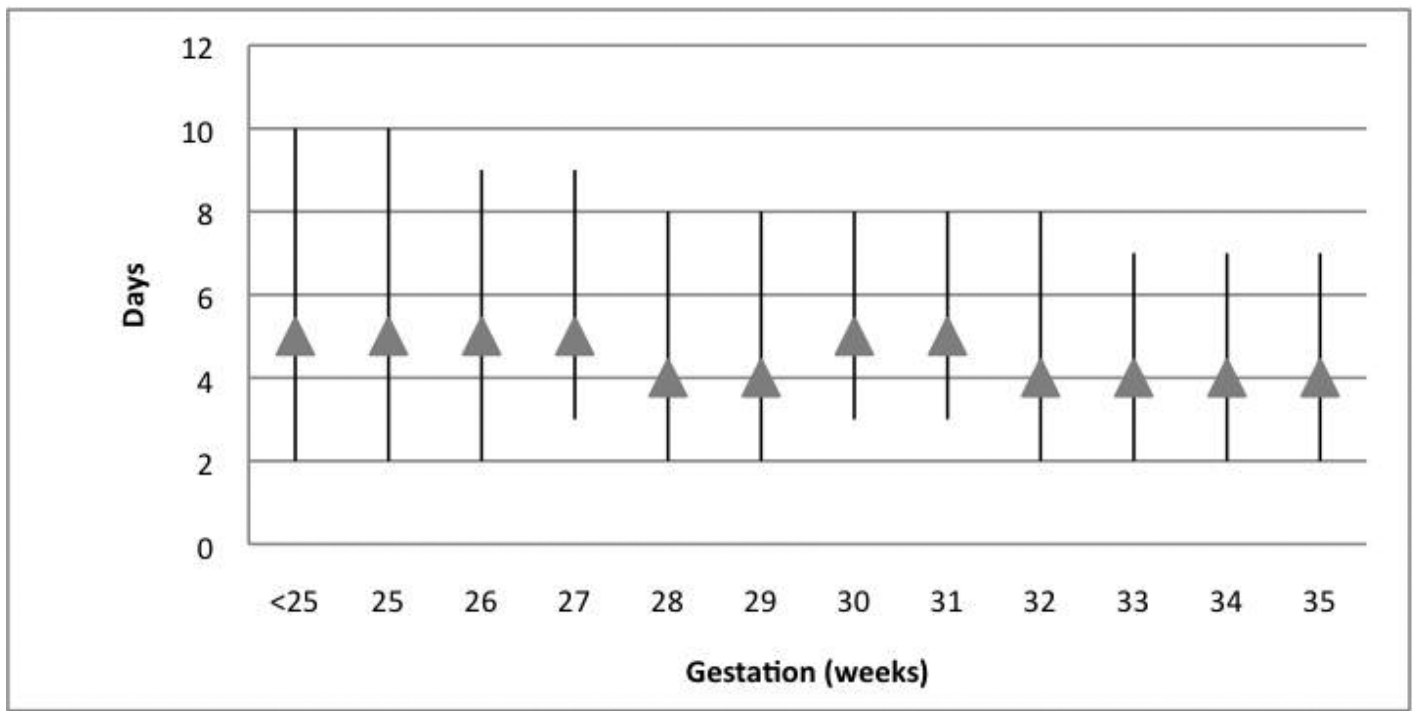

Abstract G50 Figure 3 Length of stay by gestation, median and IQR. $(p<0.05)$

in ED included thiopentone (105 patients, 42.9\%), midazolam (15, $6.12 \%)$ and midazolam and propofol (4, 1.63\%).

In ED the APLS guidelines were followed precisely in 90 patients (36.7\%); 88 patients (35.9\%) received an inappropriate dose of benzodiazepine (above guideline dose in 62, below guideline dose in 26). Thirty seven patients $(15.1 \%)$ received anticonvulsants in the wrong sequence.

The mean duration of admission to PICU was 3.7 days (range 1-45, median 2). The mean length of days ventilated (on PICU) was 3.2 days ventilated (range $1-31$, median 2 ).

Nine patients died (3.7\%). Twenty seven patients (11\%) demonstrated a new neurological deficit on discharge from PICU, of whom 10 (4\% of the entire cohort) continued to show this deficit at 30 days.
Conclusions Approximately one third of the patients admitted to PICU with RCSE had been treated in the ED appropriately using the APLS algorithm. Thiopentone was the most commonly used first-choice anticonvulsant to treat RCSE on admission to PICU. Mortality was low and approximately 1 in 25 showed a new neurological deficit at the 30-day follow-up.

\section{G52 DRUG BASED TRIAL COMPARING EFFICACY OF THREE SECOND-LINE ANTI EPILEPTIC DRUGS IN STATUS EPILEPTICUS}

doi:10.1136/archdischild-2013-304107.064

B Vyas, A Singh, S Chopra, S Shah, C Dabhi. Pediatric, M P Shah Gov Medical College, Jamnagar, India 
Background Status epilepticus is a neurological emergency and is refractory to standard treatment at times. New antiepileptic drugs have been introduced but the place of application of these drugs in status is yet to be established.

Objective To compare efficacy and adverse effects of intravenous Valproate and intravenous Levetiracetam as second line anti-epileptic drugs in status epilepticus to intravenous Phenytoin.

Methodology 42 patients between 6 months to 12 years presenting with status epilepticus were included in the study and randomly distributed in three groups depending on the day of admission and each group was assigned one of three drugs under study as second line anti-epileptic drug. The ability of the drug to stop convulsions as well as time taken to stop convulsions and adverse effects were noted. Analysis of the data was done using chi square test.

Results $57 \%$ patients studied for phenytoin(12/21), $63 \%$ for valproate $(7 / 11)$ and $80 \%$ for levitracetam $(8 / 10)$ became non convulsive after the use of respective drugs. But on applying chi-square test, $p$ value showed that the comparison was not significant. The average time taken to stop convulsions by phenytoin is $10 \mathrm{~min}$, valproate is $10 \mathrm{~min}$ and by levetiracetam is $11 \mathrm{~min}$, which again was not statistically significant. Only 2 out of $42(4.7 \%)$ developed minor adverse effects from phenytoin in the form of excessive drowsiness and irritability.

Conclusion Clinically the efficacy of i.v. levetiracetam and i.v. valproate was found to be better than i.v. phenytoin but no statistically significant difference was observed. So phenytoin remains the preferred second-line anti-convulsant in status epilepticus with minimal side-effects.

\section{G53 CURRENT PRACTISE RELATING TO FLUID BOLUS ADMINISTRATION IN CRITICALLY ILL CHILDREN}

doi:10.1136/archdischild-2013-304107.065

G Sambandamoorthy, D Inwald, M Peters, P Ramnarayan. Children's Acute Transport Service, Great Ormond Street Hospital, London, UK

Aim To establish the frequency, amount and the diagnostic reasons for fluid bolus administration in children referred to a paediatric intensive care (PIC) retrieval service

Methods Retrospective case notes review of all referrals to a PIC retrieval service between May and October 2012 (6 months). Data collected included demographic details, reason for PIC referral, and total amount of bolus fluids given at referral. Blood products were excluded from the total fluid bolus calculations. In children retrieved by the service, additional data on total amount of fluid boluses given during retrieval and patient acuity (need for invasive ventilation, vasoactive agent use and PIM-2 score) were analysed. Categorical data are reported as counts (percentages) and continuous data as mean (standard deviation) or median (inter-quartile range) as appropriate.

Results During the 6-month study period, 1031 referrals were made to the retrieval service. At referral, 180 patients had received $\geq 20 \mathrm{mls} / \mathrm{kg}$ of fluid boluses $(17.5 \%$ ) and 54 had received $\geq 40 \mathrm{mls} / \mathrm{kg}$ $(5.2 \%)$. The main diagnoses for which children were given fluid boluses $\geq 20 \mathrm{mls} / \mathrm{kg}$ were sepsis $(30 \%)$ and status epilepticus $(21 \%)$. 548 children (53\%) were retrieved to PICU. Patients receiving $\geq 40 \mathrm{mls} / \mathrm{kg}$ by PICU admission had a median age of 14 months (IOR $2-47)$, were mostly male (60\%), requiring invasive ventilation $(98.3 \%)$ and vasoactive agents (61.5\%). The median PIM-2 predicted risk of mortality was $10 \%$ (IOR 6-19\%). A summary of total fluid administered at various time points is provided below in cases where a PIC retrieval team was mobilised $(n=548)$ :

\section{Abstract G53 Table 1}

\begin{tabular}{|c|c|c|}
\hline Timepoint & $\geq 20 \mathrm{mls} / \mathrm{kg}$ & $\geq 40 \mathrm{mls} / \mathrm{kg}$ \\
\hline At referral & $141(26 \%)$ & $47(9 \%)$ \\
\hline At retrieval team arrival at local hospital & $192(35 \%)$ & $83(15 \%)$ \\
\hline At the end of retrieval & $214(39 \%)$ & $109(20 \%)$ \\
\hline
\end{tabular}

Conclusion Our audit provides valuable baseline data on the frequency, amount and timing of fluid bolus administration in critically ill children with a range of diagnoses. At a time when fluid therapy is a subject of considerable debate, this study provides useful information on current practise and may help in the design of future interventional trials in this area.

\section{G54 SHOULD HYPERNATREMIA BE TAKEN SERIOUSLY IN THE PAEDIATRIC CRITICAL CARE SETTING?}

doi:10.1136/archdischild-2013-304107.066

'D Athavale, 'M Bradbury, ${ }^{2} \mathrm{~S}$ Playfor, ${ }^{1} \mathrm{M}$ Shenoy. ${ }^{1}$ Department of Paediatric Nephrology, Royal Manchester Children's Hospital, Manchester, UK; ${ }^{2}$ Paediatric Intensive Care, Royal Manchester Children's Hospital, Manchester, UK

Aims Hypernatremia in the critical care setting is frequently observed and is shown to be associated with higher mortality in adults. We studied the prevalence and factors surrounding the presence of hypernatremia in a paediatric critical care unit.

Method From April 2007 to March 2009, the presence of hypernatremia defined as sodium $(\mathrm{Na})$ greater or equal to $150 \mathrm{mmol} / \mathrm{l}$ was studied. Demographic details of patients, including the primary intensive care unit (ICU) diagnosis were noted. The duration and peak of hypernatremia along with fluid and diuretic management was reviewed.

Results 130 episodes of hypernatremia (10\%) were detected in a total of 1301 ICU admissions over this period. Excluding 14 patients who had a head injury as cause for admission requiring active maintenance of higher sodium levels, there were 116 ICU patient episodes of hypernatremia in 104 patients (52 male, 52 female). Hypernatremia ranged from 150 - $182 \mathrm{mmol} / \mathrm{l}$ (median $155 \mathrm{mmol} / \mathrm{l}$, IOR $152-158 \mathrm{mmol} / \mathrm{l}$ ) with a duration range of 1-16 days (median 2 days, IOR 1-3 days) in the entire group. Management was variable from no active intervention to increasing fluids to administrating diuretics. ICU mortality was $4.6 \%$ (55 deaths) in the non-hypernatremic group compared to $19.0 \%$ (22 deaths) in the hypernatremic group of patients $(p<0.0001)$.

Conclusion Hypernatremia in the critical care setting is common and appears to be associated with increased mortality. Fluid management in the sick child can be challenging and the clinician needs to be alerted to initiate optimal fluid management in the hypernatremic child.

\section{G55(P) AUDIT ON OPTIMAL POSITIONING OF PERIPHERALLY INSERTED CENTRAL CATHETERS IN NEONATES}

doi:10.1136/archdischild-2013-304107.067

${ }^{1}$ A Saha, ${ }^{2} \mathrm{R}$ Fernandez. 'Department of Paediatrics, Maidstone and Tunbridge Wells Hospitals NHS Trust, Maidstone, UK; ${ }^{2}$ Trevor Mann Baby Unit, Brighton and Sussex University Hospitals NHS Trust, Brighton, UK

Aims Changes in arm position has been known to cause migration of peripherally inserted central catheter (PICC) tips in neonates, however this relationship is poorly understood. An optimal placement of central catheter tip in neonates is essential to minimise life threatening complications like cardiac tamponade.

This study aims to ascertain the relationship between arm position and central catheter tip displacement in neonates, with an aim to subsequently develop a clinical guideline on the optimal use of these lines by minimising catheter tip migration and its complications.

Methods After ethical approval was granted by the Education Department at the Deanery, an audit was undertaken at a tertiary neonatal unit to collect data on paired radiographs in order to establish the relationship between the angle of the arm at the shoulder and correlating it with the location of the catheter tip as seen on plain radiography. The angle of the arm with respect to the midline 